Retrospective Study

\title{
The Termination Level of the Dural Sac Relevant to Caudal Epidural Block in Lumbosacral Transitional Vertebrae: A Comparison between Sacralization and Lumbarization Groups
}

Ji Young Jeon, MD', Yu Mi Jeong, MD¹, Sheen-Woo Lee, MD¹, Jeong Ho Kim, MD', Hye-Young Choi, MD', and Yong Ahn, MD²

From: ${ }^{1}$ Department of Radiology, Gachon University, Gil Medical Center, Republic of Korea; ${ }^{2}$ Department of Neurosurgery, Gachon University, Gil Medical

Center, Republic of Korea

Address Correspondence: Yu Mi Jeong, MD Department of Radiology, Gachon University, Gil Medical Center

21, Namdong-daero 774 beon-gil, Namdong-gu, Incheon, 21565, Republic of Korea

E-mail:

youme34@gilhospital.com

Disclaimer: There was no external funding in the preparation of this manuscript. Conflict of interest: Each author certifies that he or she, or a member of his or her immediate family, has no commercial association (i.e., consultancies, stock ownership, equity interest, patent/licensing arrangements, etc.) that might pose a conflict of interest in connection with the submitted manuscript.

Manuscript received: 01-11-2017 Revised manuscript received: 03-29-2017 Accepted for publication: 04-16-2017

Free full manuscript: www.painphysicianjournal.com
Background: Lumbosacral transitional vertebrae (LSTV) are a relatively common variant and have been considered as one of the reasons for back pain. It is not unusual for clinicians to encounter patients with LSTV who require caudal epidural block (CEB) for pain management.

Objective: We investigated the termination level of the dural sac (DS) and anatomical features of the lumbosacral region relevant to CEB in patients with LSTV and compared these findings between sacralization and lumbarization groups.

Study Design: A retrospective evaluation.

Setting: A university hospital with inpatient and outpatient LSTV cases presenting low back pain.

Methods: Four hundred ninety-four LSTV patients were included and categorized into sacralization ( $n=201$ ) or lumbarization groups $(n=293$ ). Magnetic resonance imaging (MRI) of all of the LSTV patients were reviewed to determine the level of DS termination, the shortest distance between the apex of the sacral hiatus and DS, and the presence and the caudal level of sacral perineural cysts. Each lumbosacral vertebra column was divided into 3 equal portions (upper, middle, and lower thirds). The MRI findings in both of the groups were compared and analyzed.

Results: The distribution frequency of the levels of DS termination demonstrated a significant difference between the 2 groups. The mean caudal DS level in the lumbarization group was significantly lower than the sacralization group (lower third of the S2 [131 \{44.7\%\} of 293 patients] vs. lower third of the S1 [78 $\{38.8 \%\}$ of 201 patients]). The DS terminated at the S3 in more than $19 \%$ of the lumbarization group, whereas in only one case of the sacralization group. Although the incidence of perineural cysts was not significantly different between the 2 groups, the mean level of caudal margin of perineural cysts in the lumbarization group was significantly lower than the sacralization group (middle third of the S3 [10 $\{35.7 \%\}$ of 28 cases] vs. middle third of the S2 [11 $\{44 \%\}$ of 25 cases $])$.

Limitations: This study reveals several limitations including the practical challenge of accurate enumeration of the transitional segment and the constraints on generalizability posed by the single-country study.

Conclusion: When planning CEB for patients with LSTV, pre-procedural MRI to check the anatomical structures, including the level of DS termination and caudal margin of perineural cysts, would be of great use for lowering the risk of unexpected dural puncture during the procedure, especially in the lumbarization cases.

Key words: Termination of the dural sac, dural sac termination, lumbosacral transitional vertebrae, transitional vertebra, caudal epidural block

Pain Physician 2018; 21:73-81 
umbosacral transitional vertebrae (LSTV) are a relatively common variant and can be seen in $25 \%$ (range $15-35 \%$ ) of the general population (1-3). Morphologically, LSTV have intermediate characteristics between the sacral and the lumbar vertebrae, and the transitional segment shows an elongation of its transverse process, with varying degrees of fusion. LSTV can be defined as either sacralized L5, which means "sacralization" of the lowest lumbar segment, or lumbarized S1, which means "lumbarization" of the most superior sacral segment of the spine $(2,4)$. Back and buttock pain can be associated with LSTV, as shown by Lorenzo et al (2). Appreciation of anatomical variations due to LSTV may impact safe performance of caudal epidural steroid injection.

Caudal epidural block (CEB) is a relatively safe procedure which is used in a wide range of clinical settings $(5-8)$. It is used for intra and postoperative analgesia in a variety of operations and has become one of the most commonly performed interventions in pain practice for those with low back pain and radiculopathy. Although it is rare, the potential complication of dural puncture during CEB could occur. Therefore, determining the anatomical location of the sacral hiatus, the sacrococcygeal ligament, and the level of termination of the dural sac (DS) are essential before performing CEB, to prevent dural puncture (7-9). In this context, the questions could arise whether the termination level of the DS of LSTV patients would be significantly different from that of others with similar clinical symptoms and furthermore, within the LSTV group, the caudal level of the DS would be different based on the type of transitional segment-sacralization and lumbarization.

Although there have been many studies regarding the mean level of termination of the DS in patients with low back pain andor sciatica, there is a lack of publication that evaluated the DS termination separately for LSTV patients. Therefore, the purpose of the present study was to investigate the level of DS termination in relation to the spine in patients with LSTV, by using magnetic resonance imaging (MRI), and to compare this position between the sacralization and lumbarizaion groups. In addition, the distance between the apex of the sacral hiatus and the DS and the presence and caudal level of sacral perineural cysts were also evaluated in our LSTV patients.

\section{Methods}

This retrospective study was approved by the institutional review board and the requirement for informed consent was waived.

\section{Population}

We electronically searched the radiologic reports for MRIs of the lumbar-sacral spine that were performed from January 2000 to December 2016. The 3 keywords for the computer search were "transitional vertebra," "lumbarization," and "sacralization." This initial search selected 665 images for assessment of possible causes of lower back pain and radiculopathy. Among them, 84 images were excluded from our study owing to postoperative spine $(n=33)$, presence of severe degenerative changes $(n=31)$, such as severe scoliosis or central canal stenosis, metastasis in the lumbosacral area $(n=2)$, and in patents where identification of the DS was difficult $(n=18)$. Patients under the age of 16 years $(n=12)$ or those that did not get lumbar radiographic series-anteroposterior (AP) and lateral and with or without oblique views $(n=39)$ were excluded. Patients who did not have available plain radiographs or computed tomography (CT) images, such as radiographs of the entire spine, rib series, thoracic radiographs, or chest $\mathrm{CT}$ image, that enabled counting of the number of thoracic vertebrae and correct identification of the L1 vertebral body, were further filtered ( $n=36)$ to determine the correct numeric assignment of the LSTV. Finally, a total of 494 LSTV patients (mean age of 55.05 years, range of 17-89 years; 236 men and 258 women), composed of sacralization $(n=201)$ and lumbarization ( $n=293$ ) groups, were included in the study (Fig. 1).

\section{Image Analysis}

All images were reviewed on picture archiving and communication system workstations (G3, Infinitt, Korea). All of the 494 lumbar radiographic series were independently read by 2 radiologists (J.Y.J., 5 years of experience and Y.M.J, 8 years of experience). All LSTV patients were categorized into sacralization or lumbarization groups. Since depending on the number of thoracic vertebrae, the transitional vertebra can be interpreted as a sacralized L5 segment (sacralization) or a lumbarized S1 segment (lumbarization); we assume the number of thoracic vertebrae to be 12 in all of the patients, to gain consistency and reliability in determining the level of lumbar or sacral segment which locates the caudal tip of the DS. This would be especially helpful in confusing situations when hypoplastic ribs from transverse processes at the thoracolumbar junction or 11 and 13 pairs of ribs are seen.

Then, LSTV patients were classified according to Castellvi et al (10) (Fig. 2) into one of 4 types. Type I 
Termination Level of Dural Sac Relevant to CEB in LSTV
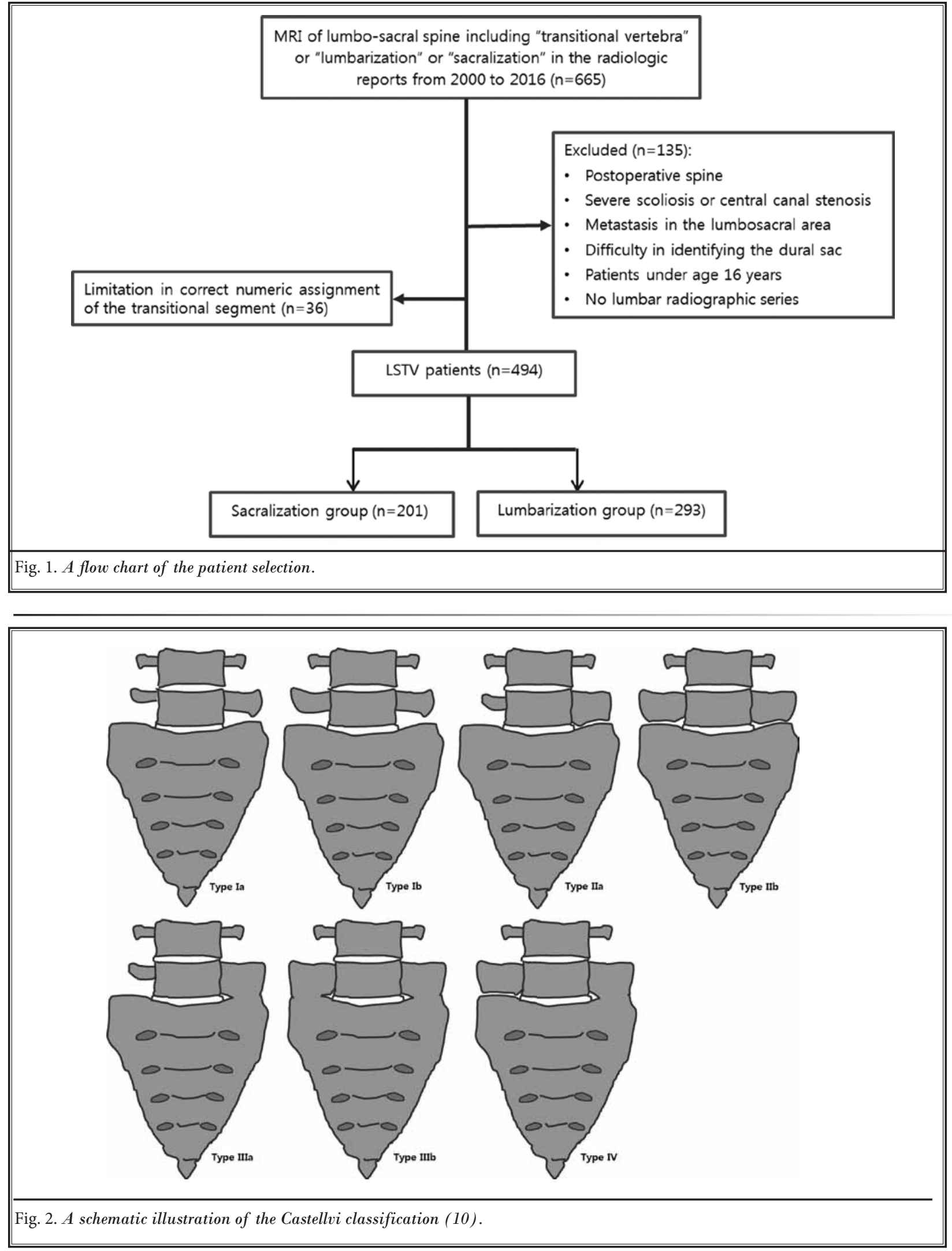
exhibits dysplastic transverse processes, measuring at least $19 \mathrm{~mm}$ in height (craniocaudal dimension). Type II includes incomplete lumbarization or sacralization with the enlarged transverse process(es) that has pseudoarthrosis with the adjacent sacral ala. Type III describes complete lumbarization or sacralization with the enlarged transverse process(es), which has complete fusion with the adjacent sacral ala. Type IV involves type II on one side and type III on the other side. To minimize the number of categories, we classified the cases into 4 basic types regardless of unilateral or bilateral findings (2). Any discrepancies were settled by mutual consensus between the authors. If there were additional available CT scans covering the entire lumbosacral junction and sacrum, the CT images were used to arbitrate a disagreement between the readers (Fig. 3).

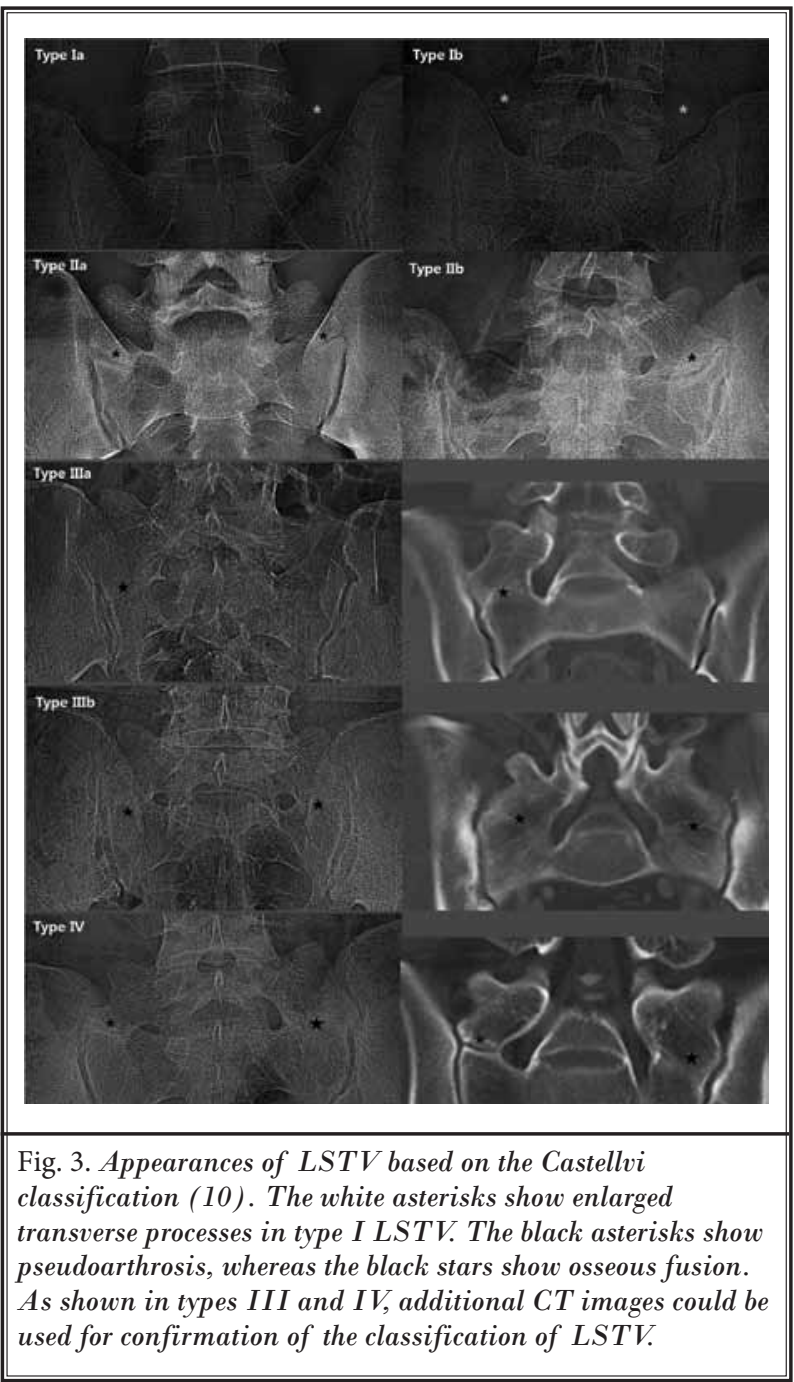

The anatomic level of the DS tip was determined by using conventional lumbar MR images. Lumbar MR images were acquired with 3.0 Tesla (T) MRI machines (Skyra and Verio, Siemens Healthcare, Erlangen, Germany) or 1.5-T scanners (Avanto, Siemens Healthcare, Erlangen, Germany) using phased array spine coils in a supine position. Sagittal images were acquired using a slice thickness of $3.0 \mathrm{~mm}$ and a $0.3 \mathrm{~mm}$ interslice gap. Routine scans consisted of T1-weighted sagittal and axial images (TE range $3-13 \mathrm{~ms}$, TR range $450-704 \mathrm{~ms}$ ) and T2-weighted sagittal and axial images (TE range 91 - 95ms, TR range 3200 - 5910ms). In some MRI studies, sagittal short-tau inversion recovery (STIR) sequence (TE range $55-81 \mathrm{~ms}$, TR range $2500-4310 \mathrm{~ms}$ ) of the whole spine was available. The midsagittal sections for each subject were used when possible to improve the reliability for the measurement of the caudal end of DS.

Two radiologists independently reviewed the MR images. To establish the caudal tip of the DS in relation to the lumbosacral spine, the $L 5$ and each sacral vertebra column was divided into 3 equal portions (upper, middle, and lower thirds [u, m, and l, respectively]) in considering the method of Saifuddin et al (11) (Fig. 4). By extending a line perpendicular to the long axis of the dura at the most inferior convergence point of the DS, the point where the perpendicular line crosses the adjacent vertebrae was determined to be the most caudal level of the DS. For statistical evaluation, each lumbosacral vertebral regional level of the caudal DS tip was assigned as numbers from one (lower third of the L5) to 10 (lower third of the S3). The shortest distance between the upper margin of the sacrococcygeal membrane and the DS was measured to estimate the safe length of advancing the needle for CEB (Fig. 5). The presence of a perineural cyst (Tarlov cyst) and its caudal level were also investigated (8). The level of caudal margin of perineural cysts were assigned as numbers in the same manner as described above. In cases where there was a disagreement between the readers, further discussion had been made to achieve consensus.

\section{Statistical Analysis}

All statistical analysis was performed using commercial software SPSS Version 20.0 (IBM Corporation, Armonk, NY). Descriptive statistics for baseline demographic data were calculated. Continuous variables are presented as mean \pm standard deviation and categorical variables as frequency (percentages). For quantitative and qualitative comparisons between the 2 LSTV groups with lumbarization and sacralization, continu- 


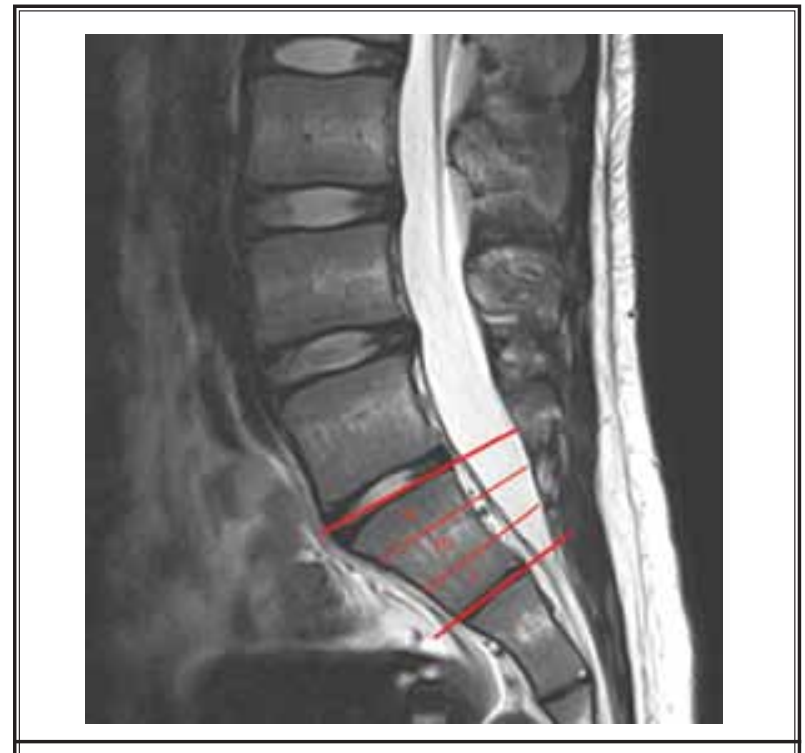

Fig. 4. The midsagittal section of a MR image of the lumbosacral spine demonstrating our method for determining the position of termination of the DS in relation to the lumbosacral vertebrae (u: upper third, $m$ : middle third, $l$ : lower third).

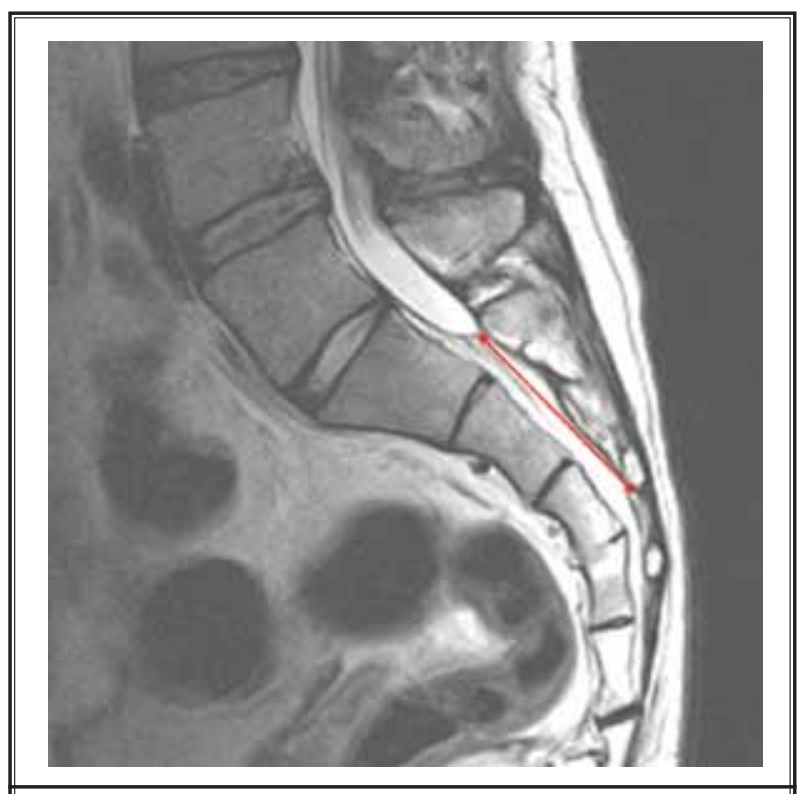

Fig. 5. The midsagittal section of a MR image of the lumbosacral spine showing how the distance (double arrow) between the upper margin of the SCM and the DS was measured. ous variables were compared by using the analysis of an independent samples t-test, and categorical variables were compared by using a chi-square test. For all tests, $P<0.05$ was considered to be indicative of a statistically significant difference.

\section{Results}

Demographic data and cross-tabulation of imaging findings, other than the level of DS termination of all LSTV patients $(n=494)$, sacralization group $(n=201)$, and lumbarization group ( $n=293)$, are outlined in Table 1.

No significant difference was found in age between the sacralization and lumbarization groups ( $P$ $=.364$ ). There were significantly more women (133 of 201) than men (68 of 201) in the sacralization group, whereas there were more men (168 of 293) than women (125 of 293) in the lumbarization group $(P<$ .001). The 494 patients with LSTV were placed into 4 Castellvi classification subgroups: 101 patients (20.4\%) had LSTV type I, 194 patients (39.3\%) had LSTV type II, 141 patients $(28.5 \%)$ had LSTV type III, and 58 patients $(11.7 \%)$ had LSTV type IV. LSTV type I was significantly more common in the lumbarization group than in the sacralization group (92 [31.4\%] of 293 patients vs. 9
[4.5\%] of 201 patients, $P<.001)$, whereas LSTV type III and IV were significantly more common in the sacralization group (84 [41.8\%] of 201 patients for type III and 36 [17.9\%] of 201 for type IV) than in the lumbarization group (57 [19.5\%] of 293 patients for type III and 22 [7.5\%] of 293 for type IV) $(P \leq .001)$.

In our study population, 53 cases (25 [12.4\%] of the sacralization group and 28 [9.6\%] of the lumbarization group) of sacral perineural cysts were seen. The incidence of perineural cysts was not significantly different between the 2 groups $(P=.375)$ (Table 1).

The mean shortest linear distance between the upper margin of the sacrococcygeal membrane (SCM) and the DS showed no significant difference between the sacralization (31.1 $\mathrm{mm}$ ) and lumbarization groups (27.9 $\mathrm{mm})(P=.232)$ (Table 1).

The level of DS termination was extending from the lower third of the L5 to the lower third of the S3. The level of termination of the DS in all of the LSTV patients was most commonly the lower one-third of the S2 (in $26.9 \%$ of the total population). In comparing by groups, however, the distribution frequency of the levels of DS termination on MR images demonstrated a significant difference between the sacralization and lumbarization groups. The mean caudal DS level in the 
Table 1. Demographic data and results of cross-tabulation of findings

\begin{tabular}{|l|c|c|c|c||}
\hline \multicolumn{1}{|c|}{} & $\begin{array}{c}\text { Total } \\
(\mathbf{n = 4 9 4 )}\end{array}$ & $\begin{array}{c}\text { Sacralization Group } \\
(\mathbf{n}=\mathbf{2 0 1})\end{array}$ & $\begin{array}{c}\text { Lumbarization Group } \\
(\mathbf{n}=\mathbf{2 9 3})\end{array}$ & $\boldsymbol{P}$-value \\
\hline Age (years)* & $55 \pm 17.8$ & $53.7 \pm 18.2$ & $55.9 \pm 17.6$ & 0.364 \\
\hline Gender & & & 168 & $<0.001$ \\
\hline Male & 236 & 133 & 125 & \\
\hline Female & 258 & & $92(31.4)$ & $<0.001$ \\
\hline Subtype & & $9(4.5)$ & $122(41.6)$ & 0.223 \\
\hline LSTV type I & $101(20.4)$ & $72(35.8)$ & $57(19.5)$ & $<0.001$ \\
\hline LSTV type II & $194(39.3)$ & $84(41.8)$ & $22(7.5)$ & 0.001 \\
\hline LSTV type III & $141(28.5)$ & $36(17.9)$ & & 0.375 \\
\hline LSTV type IV & $58(11.7)$ & & 28 & 265 \\
\hline Perineural Cyst & & 25 & $27.9 \pm 24.4$ & 0.232 \\
\hline Presence & 53 & $31.1 \pm 24.5$ & & \\
\hline Absence & $29.1 \pm 24.5$ & & & \\
\hline $\begin{array}{l}\text { Shortest Distance Between SCM } \\
\text { and DS (mm)* }\end{array}$ & & & & \\
\hline
\end{tabular}

LSTV = lumbosacral transitional vertebrae, SCM = sacrococcygeal membrane, DS = dural sac

${ }^{\star}$ Data are the mean \pm standard deviation

Table 2. Incidence of caudal level of the DS in LSTV patients and comparison between the sacralization and lumbarization groups

\begin{tabular}{|c|c|c|c|c|}
\hline \multirow[b]{2}{*}{ Caudal Level of DS } & \multirow[b]{2}{*}{ Total $(n=494)$} & \multicolumn{2}{|c|}{ Number of LSTV Patients (\%) } & \multirow[b]{2}{*}{$P$-value } \\
\hline & & $\begin{array}{l}\text { Sacralization Group } \\
(\mathbf{n}=201)\end{array}$ & $\begin{array}{c}\text { Lumbarization Group } \\
(\mathrm{n}=\mathbf{2 9 3})\end{array}$ & \\
\hline Lower third of L5 & $5(1)$ & $5(2.5)$ & $0(0)$ & 0.011 \\
\hline Upper third of S1 & $22(4.5)$ & $22(10.9)$ & $0(0)$ & $<0.001$ \\
\hline Middle third of S1 & $48(9.7)$ & $47(23.4)$ & $1(0.3)$ & $<0.001$ \\
\hline Lower third of S1 & $83(16.8)$ & $78(38.8)$ & $5(1.7)$ & $<0.001$ \\
\hline Upper third of S2 & $75(15.2)$ & $38(18.9)$ & $37(12.6)$ & 0.073 \\
\hline Middle third of S2 & $71(14.4)$ & $8(4.0)$ & $63(21.5)$ & $<0.001$ \\
\hline Lower third of S2 & $133(26.9)$ & $2(1.0)$ & $131(44.7)$ & $<0.001$ \\
\hline Upper third of S3 & $43(8.7)$ & $1(0.5)$ & $42(14.3)$ & $<0.001$ \\
\hline Middle third of S3 & $12(2.4)$ & $0(0)$ & $12(4.1)$ & 0.002 \\
\hline Lower third of S3 & $2(0.4)$ & $0(0)$ & $2(0.7)$ & 0.516 \\
\hline
\end{tabular}

LSTV = lumbosacral transitional vertebrae, DS = dural sac

lumbarization group was significantly lower than the sacralization group (lower third of the S2 [131 \{44.7\%\} of 293 patients] vs. lower third of the S1 [78 \{38.8\%\} of 201 patients], $P<.001)$. The caudal end of the DS terminated above the S2 vertebra in only $2 \%$ of the lumbarization group (6 of 293 patients), while in more than $75 \%$ of the sacralization group (152 [75.6\%] of 201 patients). The DS was found to terminate at the S3 in more than $19 \%$ of the lumbarization group (56 [19.1\%] of 293 patients), whereas in only one case of the sacralization group (one [0.5\%] of 201 patients) (Table 2) (Fig. 6).
The mean level of caudal margin of perineural cysts in the lumbarization group was significantly lower than in the sacralization group (middle third of the S3 [10 $\{35.7 \%\}$ of 28 cases] vs. middle third of the S2 [11 $\{44 \%\}$ of 25 cases], $P \leq .001$ ) (Table 3 ).

\section{Discussion}

It has been known that back pain correlates with LSTV $(2,12-15)$. Therefore, it is not uncommon to encounter patients with LSTV in the process of back and buttock pain management such as epidural steroid injection or epidural adhesiolysis. Although the cau- 
dal approach to the epidural space is a relatively safe procedure, extension of the dura to a lower-than-expected sacral level as a simple anatomic variant could increase the risk of dural puncture or inadvertent spinal anesthesia during procedure $(6,8,16)$. Hence, our study investigated the level of termination of the DS in a group of patients with LSTV and demonstrated that the mean level DS termination was significantly lower in the lumbarization group than in the sacralization group.

We found that the level of DS termination in all of the LSTV patients was most commonly the lower third of the S2. This finding is in agreement with previous cadaveric studies $(17,18)$ and MRI studies with the living population, revealing that the mean level of the DS tip in adults lies at the lower border of the S2 $(19,20)$. In our study, the caudal tip of the DS was found at the S3 in about $19 \%$ of cases of the lumbarization group, with a higher rate than earlier reports by Aggarwal et al (9), Cilliers et al (21), and Senoglu et al (8). It has been reported that the termination of the DS at the S3 might result in a higher rate of dural puncture than at the S2 $(6,8,9)$. Therefore, more caution should be taken to the LSTV patients with lumbarization when using the caudal approach.

Since cystic structures such as posterior sacral meningoceles or perineural cysts extended to the lower sacrum could cause unexpected dural puncture $(5,6,22)$, we also investigated the prevalence of these cystic structures in our LSTV population. Perineural cysts were seen in about $10 \%$ of all patients and no cases of posterior sacral meningoceles were seen. The higher prevalence of perineural cysts in our study $(10 \%)$ compared to previous studies $(1.3-4.6 \%)(6,8,22)$ may be partly explained by a difference in the patient population selected for the studies, seeing as this study was designed based on LSTV cases. As expected, the mean level of caudal margin of perineural cysts in

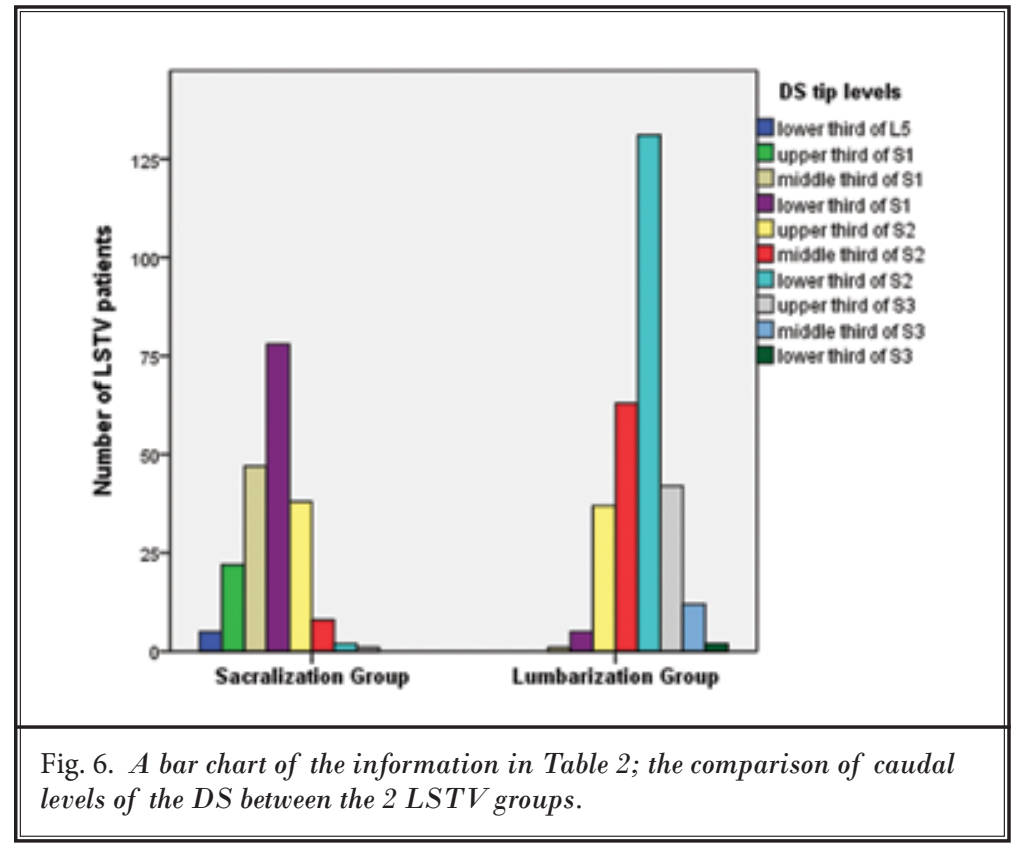

Table 3. The vertebral level of caudal margin of perineural cysts, seen in 53 of 494 LSTV patients

\begin{tabular}{|l|c|c|c|}
\hline \multirow{2}{*}{$\begin{array}{c}\text { Caudal Margin } \\
\text { of Perineural } \\
\text { Cysts }\end{array}$} & \multicolumn{2}{|c|}{ LSTV Group } & \multirow{2}{*}{ P-value } \\
\cline { 2 - 3 } & $\begin{array}{c}\text { Sacralization Group } \\
(\mathbf{n}=\mathbf{2 5})\end{array}$ & $\begin{array}{c}\text { Lumbarization Group } \\
(\mathbf{n}=\mathbf{2 8})\end{array}$ & \\
\hline Lower third of S1 & $1(4.0)$ & $0(0)$ & 0.285 \\
\hline Upper third of S2 & $9(36.0)$ & $2(7.1)$ & 0.016 \\
\hline Middle third of S2 & $11(44.0)$ & $0(0)$ & $<0.001$ \\
\hline Lower third of S2 & $3(12.0)$ & $4(14.3)$ & 1.000 \\
\hline Upper third of S3 & $1(4.0)$ & $6(21.4)$ & 0.104 \\
\hline Middle third of S3 & $0(0)$ & $10(35.7)$ & 0.001 \\
\hline Lower third of S3 & $0(0)$ & $6(21.4)$ & 0.016 \\
\hline \hline
\end{tabular}

LSTV = lumbosacral transitional vertebrae

the lumbarization group was significantly lower than in the sacralization group, since the DS terminal was located more distally in the former group. However, unlike previous reports, perineural cysts below the S3 were not found in any group of LSTV patients $(6,8)$.

With regards to the type of LSTV, the incidence of each subtype of LSTV was significantly different between groups. In particular, the majority of type 1 LSTV patients was found in the lumbarization group, therefore we could assume that sacralization of transitional vertebra is more inclined to form pseudoarthrosis or complete osseous fusion with the sacral ala.

During the caudal approach, it is also necessary to know the distance between the apex of the hiatus and the end of the DS for deter- 
mining the length of the needle to be introduced into the canal $(5,7,8)$. Aggarwal et al $(9)$ found the mean distance between the apex of the hiatus and the end of the DS to be $31.6 \pm 12 \mathrm{~mm}$ (range $5.76-60.0 \mathrm{~mm}$ ). We found a similar mean distance of $29.1 \mathrm{~mm}$, considering measurement error, with a similarly large range of distances $3.7-85 \mathrm{~mm}$. Since the minimum value was less than $5 \mathrm{~mm}$, the needle should be advanced carefully from the sacrococcygeal ligament during a caudal approach to prevent dural puncture in patients with LSTV, as applied to other patients.

However, there are several limitations to the current study. First, it is possible that some cases of LSTV could be missed because we had performed text searches of radiologic reports of lumbosacral MRI for the initial patient selection. These missing data might have influenced the results. Second, for accurate numbering of a transitional segment, we selected all LSTV cases that were possible to identify the entire thoracic segments and to correctly assign the $L 1$ vertebral body - for example, patients who had radiographs of the whole spine or thoracic spine. However, in local clinics, as it is more common to have lumbar spine radiographs alone without other available imaging, accurate enumeration of the transitional segments could not always be possible. Third, since this was a single-center, single-country study, it may have limited the generalizability. Multi-center, multi-country studies with a larger number of LSTV cases are warranted to consolidate our findings. Fourth, in the subtype classifications, we did not make a distinction between unilateral and bilateral pseudoarthrosis and fusion. Since it is beyond our scope to compare the difference of LSTV subtypes seen in the sacralization and lumbarization groups, it was unnecessary to make the model complicated by increasing the 4-level outcome to an 8-level outcome. Finally, measures of interrater agreement were not obtained about imaging data; however, analysis of the raw data did not reveal a big difference between the readers.

\section{Conclusion}

Consequently, as the main result, our study demonstrated a difference in the DS termination level between groups of transitional vertebra with lumbarizaion and sacralization, which means that the position of the DS tip in the lumbarization group was significantly lower than in the sacralization group, and in the lumbarization group, the proportion of cases that the DS tip was located at the S3 was greater than that in the previously reported general population studies. Therefore, when planning caudal procedures for pain management in patients with LSTV, especially in the lumbarization cases, pre-procedural MR imaging to check the termination level of the DS, the distance between the upper margin of the SCM and the end of the DS, and the presence of incidental cystic structures would be of great use for lowering the risk of unexpected dural puncture during the procedure.

\section{References}

1. Carrino JA, Campbell PD Jr, Lin DC, Morrison WB, Schweitzer ME, Flanders $A E$, Eng J, Vaccaro AR. Effect of spinal segment variants on numbering vertebral levels at lumbar MR imaging. Radiology 2011; 259:196-202.

2. Nardo L, Alizai $H$, Virayavanich W, Liu F, Hernandez A, Lynch JA, Nevitt MC, McCulloch CE, Lane NE, Link TM. Lumbosacral transitional vertebrae: Association with low back pain. Radiology 2012; 265:497-503.

3. Tini PG, Wieser C, Zinn WM. The transitional vertebra of the lumbosacral spine: Its radiological classification, incidence, prevalence, and clinical significance. Rheumatol Rehabil 1977; 16:180-185.

4. Hughes RJ, Saifuddin A. Imaging of lumbosacral transitional vertebrae. Clin Radiol 2004; 59:984-991.
5. Park BC, Kim BS, Hwang WJ, Lee J, Moon DE. Anatomical variations which can result in inadvertent dural puncture when performing caudal block: A report of 3 cases. Korean J Anesthesiol 2006; 50:332-336.

6. Joo J, Kim J, Lee J. The prevalence of anatomical variations that can cause inadvertent dural puncture when performing caudal block in Koreans: A study using magnetic resonance imaging. Anaesthesia 2010; 65:23-26.

7. Kim SG, Yang JY, Kim DW, Lee YJ. Inadvertent dural puncture during caudal approach by the introducer needle for epidural adhesiolysis caused by anatomical variation. Korean J Pain 2013; 26:203-206.

8. Senoglu N, Senoglu M, Ozkan F, Kesilmez $C$. The level of termination of the dural sac by MRI and its clinical rele- vance in caudal epidural block in adults. Surg Radiol Anat 2013 35:579-584.

9. Aggarwal A, Kaur H, Batra YK, Aggarwal AK, Rajeev S, Sahni D. Anatomic consideration of caudal epidural space: A cadaver study. Clin Anat 2009; 22:730-737.

10. Castellvi AE, Goldstein LA, Chan DP. Lumbosacral transitional vertebrae and their relationship with lumbar extradural defects. Spine (Phila Pa 1976) 1984; 9:493-495.

11. Saifuddin A, Burnett SJ, White J. The variation of position of the conus medullaris in an adult population: $A$ magnetic resonance imaging study. Spine (Phila Pa 1976) 1998; 23:1452-1456.

12. Luoma K, Vehmas T, Raininko R, Luukkonen R, Riihimäki $H$. Lumbosacral transitional vertebra: Relation to disc degeneration and low back pain. Spine 
(Phila Pa 1976) 2004; 29:200-205.

13. Taskaynatan MA, Izci Y, Ozgul A, Hazneci $B$, Dursun $H$, Kalyon TA. Clinical significance of congenital lumbosacral malformations in young male population with prolonged low back pain. Spine (Phila Pa 1976) 2005; 30:E210-E213.

14. Marks RC, Thulbourne T. Infiltration of anomalous lumbosacral articulations: Steroid and anesthetic injections in 10 back-pain patients. Acta Orthop Scand 2009; 62:139-141.

15. Oyinloye OI, Abdulkadir AY, Babalola OM. Incidence and patterns of lumbosacral transitional vertebrae, in patients with low backpain in a Nigerian hospi- tal. Nig QJ Hosp Med 2009; 19:95-99.

16. Crighton IM, Barry BP, Hobbs GJ. A study of the anatomy of the caudal space using magnetic resonance imaging. Br J Anaesth 1997; 78:391-395.

17. McCotter RE. Regarding the length and extent of the human medulla spinalis. Anat Rec 1916; 10:559-564.

18. Needles $\mathrm{JH}$. The caudal level of termination of the spinal cord in American whites and American negroes. Anat Rec 1935; 63:417-424.

19. Macdonald A, Chatrath P, Spector T, Ellis $H$. Level of termination of the spinal cord and the dural sac: A magnetic resonance study. Clin Anat 1999; 12:149-152.
20. Standring S. Gray's Anatomy. The Anatomical Basis of Clinical Practice. 40th ed. Churchill Livingstone, Philadelphia. 2008, pp 762-774.

21. Cilliers A, Schulenburg DH, van Rensburg J. MRI determination of the vertebral termination of the dural sac tip in a South African population: Clinical significance during spinal irradiation and caudal anaesthesia. SA Journal of Radiology 2010; 14:52.

22. Lucantoni C, Than KD, Wang AC, Valdivia-Valdivia JM, Maher CO, La Marca F, Park P. Tarlov cysts: A controversial lesion of the sacral spine. Neurosurg Focus 2011; 31:E14. 
